\title{
A CONVERSE OF THE VOLUME-MEAN-VALUE PROPERTY FOR INVARIANTLY HARMONIC FUNCTIONS
}

\author{
JOAQUIM BRUNA AND JACQUELINE DETRAZ
}

(Communicated by Eric Bedford)

\begin{abstract}
It is shown that the balls are the only domains having the mean value property with respect to the invariantly harmonic functions in the unit ball of $\mathbb{C}^{n}$.
\end{abstract}

\section{INTRODUCTION}

Let $B$ be the unit ball in $\mathbb{C}^{n}$. The Laplacian with respect to the Bergman metric in $B$ is given in coordinates by

$$
\Delta=\left(1-|z|^{2}\right) \sum_{i, j=1}^{n}\left(\delta_{i j}-z_{i} \bar{z}_{j}\right) D_{i} \bar{D}_{j}
$$

It is also called the invariant Laplacian because for every $f \in C^{2}(B)$ and every complex automorphism $\Psi$ of $B$ one has $\Delta(f \circ \Psi)=(\Delta f) \circ \Psi$. The group $\operatorname{Aut}(B)$ of such automorphisms is generated by the unitary operators on $\mathbb{C}^{n}$ and the involutions $\varphi_{a}$ permuting $a$ and 0 . The functions $f$ such that $\Delta f=0$ are called $\mathscr{M}$-harmonic (we refer to [7, Chapter 4] for general properties of these functions).

If $f$ is $\mathscr{M}$-harmonic in $B$, the mean-value of $f$ on a sphere centered at 0 is $f(0)$ [7, p. 51]. Therefore, if $B_{r}$ denotes the Euclidean ball of radius $r$ centered at 0 and $d m$ denotes normalized Lebesgue measure in $B$, for a measure $d \mu$ of type $d \mu=\varphi(|\zeta|) d m(\zeta)$ one has

$$
f(0)=\frac{1}{\mu\left(B_{r}\right)} \int_{B_{r}} f d \mu
$$

for all $f \mathscr{M}$-harmonic in $B$ (in fact, for all $f \mathscr{M}$-harmonic and $d \mu$-integrable in $B_{r}$ ).

This is the case for the measure $d \lambda=\left(1-|\zeta|^{2}\right)^{-n-1} d m$. The measure $d \lambda$ is invariant under the action of $\operatorname{Aut}(B)$, whence

$$
f(a)=\frac{1}{\lambda\left(B_{r}\right)} \int_{\varphi_{a}\left(B_{r}\right)} f(\zeta) d \lambda(\zeta)
$$

Received by the editors March 1, 1993.

1991 Mathematics Subject Classification. Primary 32A99, 31 B05.

Key words and phrases. Invariantly harmonic functions, Green function, Bergman Laplacian.

The first author was partially supported by DGICYT grant PB92-0804-C02. 
for all $f \mathscr{M}$-harmonic and $d m$-integrable in $\varphi_{a}\left(B_{r}\right)$; i.e., $f$ has the mean value property with respect to $d \lambda$ and the "Bergman balls" $\varphi_{a}\left(B_{r}\right)$. In this paper we prove a sort of converse of this fact:

Theorem 1. Let $U$ be open, connected, relatively compact in $B$, and such that $\partial U=\partial \bar{U}$. Assume $a \in U$ is such that

$$
f(a)=\frac{1}{\lambda(U)} \int_{U} f(\zeta) d \lambda(\zeta)
$$

for all $\mathscr{M}$-harmonic functions $f$ in a neighbourhood of $\bar{U}$. Then $U$ is a Bergman ball centered at a; i.e., $U=\varphi_{a}\left(B_{r}\right)$ for some $r<1$.

The analogue of this theorem for harmonic functions in Euclidean space (and Euclidean balls) was proved in [4] (see also [5] and [2]). See [1] for a converse, in another sense, of the mean-value property $(*)$.

\section{THE PROOF}

We need some known facts about $\mathscr{M}$-harmonic functions that we will proceed to recall. There is a Riesz-type decomposition formula, valid at least for $u \in$ $C^{2}(\bar{B})$

$$
u(z)=\int_{S} P(\zeta, z) u(\zeta) d \sigma(\zeta)+\int_{B} \Delta u(\zeta) G(\zeta, z) d \lambda(\zeta), \quad z \in B
$$

Here $S=\partial B, d \sigma$ is the normalized Lebesgue measure on $S, P(\zeta, z)$ is the invariant (or Poisson-Szegö) kernel, and $G(\zeta, z)$ is the Green function with pole at $z$,

$$
G(\zeta, z)=g\left(\left|\varphi_{z}(\zeta)\right|^{2}\right), \quad g(x)=-c_{n} \int_{x}^{1} \frac{(1-t)^{n-1}}{t^{n}} d t,
$$

$c_{n}$ being a positive constant. Moreover,

$$
1-\left|\varphi_{z}(\zeta)\right|^{2}=\frac{\left(1-|z|^{2}\right)\left(1-|\zeta|^{2}\right)}{|1-\bar{\zeta} z|^{2}},
$$

so $G$ is symmetric. From the formula it follows that if $f$ has compact support and is $\mathscr{M}$-harmonic in $\bar{U}$, then

$$
f(z)=\int_{B \backslash U} \Delta f(\zeta) G(\zeta, z) d \lambda(\zeta), \quad z \in U
$$

This means that the functions $G_{z}(\zeta)=G(\zeta, z), z \notin \bar{U}$, span the space of all $\mathscr{M}$-harmonic functions in a neighbourhood of $\bar{U}$. It also follows from the formula that under suitable conditions on $\Psi$, the Green potential

$$
G \Psi(z)=\int_{B} \Psi(\zeta) G(\zeta, z) d \lambda(\zeta)
$$

satisfies $\Delta G \Psi=\Psi$ in the weak sense.

Let us begin the proof of the theorem. Obviously we can assume $a=0$. By what has been said, the hypothesis is equivalent to

$$
c G(0, z)=\int_{U} G(\zeta, z) d \lambda(\zeta), \quad z \notin \bar{U},
$$


with $c=\lambda(U)$. Let us look at the potential

$$
h(z)=\int_{U} G(\zeta, z) d \lambda(\zeta), \quad z \in B
$$

We know that $h(z)=c G(0, z)$ for $z \notin \bar{U}$. In $U, h$ satisfies $\Delta h=1$; a computation shows that $\frac{1}{n} \log \left(1-|z|^{2}\right)$ has invariant Laplacian 1 in $B$, hence

$$
h(z)=\frac{1}{n} \log \frac{1}{1-|z|^{2}}+u(z), \quad z \in U,
$$

with $u \mathscr{M}$-harmonic in $U$.

Lemma 1. $h$ is of class $C^{1}$ in $B$.

Proof. One must prove that $\nabla_{z} G(\zeta, z)$ is locally uniformly integrable. Using the formulas above,

$$
\begin{aligned}
\left|D_{i} G(\zeta, z)\right|= & c_{n} \frac{\left(1-\left|\varphi_{z}(\zeta)\right|^{2}\right)^{n-1}}{\left|\varphi_{z}(\zeta)\right|^{2 n}} \frac{1-|\zeta|^{2}}{|1-\zeta \bar{z}||1-\bar{\zeta} z|^{2}} \\
& \times\left|\bar{\zeta}_{i}\left(1-|z|^{2}\right)-\bar{z}_{i}(1-\bar{\zeta} z)\right| \\
= & c_{n} \frac{\left(1-|z|^{2}\right)^{n-1}\left(1-|\zeta|^{2}\right)^{n-1}}{\left[|1-\bar{\zeta} z|^{2}-\left(1-|z|^{2}\right)\left(1-|\zeta|^{2}\right)\right]^{n}} \frac{1-|\zeta|^{2}}{|1-\zeta \bar{z}|} \\
& \times\left|\bar{\zeta}_{i}\left(1-|z|^{2}\right)-\bar{z}_{i}(1-\bar{\zeta} z)\right| .
\end{aligned}
$$

Therefore,

$$
\mid \nabla_{z} G(\zeta, z) \leq C \frac{|\zeta-z|}{\left[|1-\bar{\zeta} z|^{2}-\left(1-|z|^{2}\right)\left(1-|\zeta|^{2}\right)\right]^{n}}, \quad \zeta, z \in B
$$

This is a well-known singularity; in fact, it is the same that appears in the Cauchy kernel for the ball. If $e_{1}, \ldots, e_{n-1}$ is an orthonormal basis of $\left(\mathbb{C}_{z}\right)^{\perp}$ and $\zeta-z=\lambda_{n} z+\sum \lambda_{i} e_{i}$, the expression inside the brackets equals

$$
\left|\lambda_{n}\right|^{2}|z|^{2}+\left(1-|z|^{2}\right) \sum_{i=1}^{n-1}\left|\lambda_{i}\right|^{2} \geq\left(1-|z|^{2}\right)|\zeta-z|^{2}
$$

Hence, for $z$ in a compact set $K \subset B$,

$$
\left|\nabla_{z} G(\zeta, z)\right| \leq C_{K}|\zeta-z|^{1-2 n},
$$

which proves the lemma.

By Lemma $1, u \in C^{1}(\bar{U})$, and since $\partial U=\partial \bar{U}$, it follows that $u$ coincides on $\partial U$ with

$$
c G(0, z)-\frac{1}{n} \log \frac{1}{1-|z|^{2}}
$$

a radial function, up to order one.

Our objective is to prove that $u$ is constant. At this point, we need to introduce the tangent fields

$$
R=\sum_{1}^{n} z_{j} D_{j}=N+i T, L_{j k}=\bar{z}_{j} D_{k}-\bar{z}_{k} D_{j}, \quad j \neq k
$$


The fields $T, \operatorname{Re} L_{j k}$, and $\operatorname{Im} L_{j k}$ span at each point $z$ the tangent space to the sphere of radius $|z|$. We write

$$
\Delta_{0}=\sum_{i, j=1}^{n}\left(\delta_{i j}-z_{i} \bar{z}_{j}\right) D_{i} \bar{D}_{j} .
$$

By direct computation one proves

Lemma 2. (a) $\Delta_{0} T-T \Delta_{0}=0$.

(b) If $L$ denotes one of the $L_{j k}, \Delta_{0} L-L \Delta_{0}=2 i L T$.

By Lemma 2(a), $T u$ is also $\mathscr{M}$-harmonic in $U$. But $u$ is radial on $\partial U$ up to order 1 ; hence, $T u=0$ on $\partial U$. By the maximum principle for $\mathscr{M}$-harmonic functions, $T u \equiv 0$ in $U$.

Lemma 2(b) implies then that each $L_{j k} u$ is also $\mathscr{M}$-harmonic in $U$. As $u$ is radial on $\partial U$ up to order 1 , it follows as before that $L_{j k} u \equiv 0$ in $U$ for all $j, k$.

We have thus proved that

$$
T u=L_{j k} u=0 \text { in } U \text {. }
$$

Let $B^{\prime}$ be a ball centered at 0 included in $U$; then $u$ is radial in $B^{\prime}$. Being $\mathscr{M}$-harmonic in $B^{\prime}$ and radial, it must be constant. By analytic continuation, $u$ is constant, say $k$, in $U$.

Then

$$
c g\left(|z|^{2}\right)-\frac{1}{n} \log \frac{1}{1-|z|^{2}}=k, \quad z \in \partial U .
$$

The left-hand side, as a function of $|z|$, increases from $-\infty$ to a maximum value and then decreases again to $-\infty$ when $|z| \rightarrow 1$. Therefore, $|z|$ takes at most two values on $\partial U$, and since $U$ was assumed to contain $0, U$ must be a ball centered at 0 .

\section{REMARKS AND QUESTIONS}

(a) The following approximation theorem can be obtained by combining a general result of [6] on the Runge property for functions annilated by analytichypoelliptic operators and [7, 5.5.4] (see also [3] for a different proof).

Theorem 2. Assume $K$ is a compact set in $B$ such that $B \backslash K$ is connected. Then every $\mathscr{M}$-harmonic function $f$ in a neighbourhood of $K$ is the uniform limit on $K$ of a sequence of $\mathscr{M}$-harmonic functions in the whole ball $B$ and continuous on $\bar{B}$.

Hence if $U$ is as in the theorem and, moreover, $B \backslash \bar{U}$ is connected, then from the assumption

$$
f(a)=\frac{1}{\lambda(U)} \int_{U} f d \lambda
$$

for all $f \mathscr{M}$-harmonic in $B$ and continuous on $\bar{B}$ it follows that $U$ is a Bergman ball centered at $a$. We do not know if this holds true when $B \backslash \bar{U}$ is not connected. 
In the case of harmonic functions on Euclidean space, Shapiro [8] provided us an example of a (doubly connected) open set $A$ of $\mathbb{R}^{2}$ such that $0 \in A$, $\int_{A} z^{n} d m(z)=0 \quad \forall n \geq 1$, and $A$ is not a disc centered at 0 .

(b) The same method proves the following result: let $U$ be as in the theorem, $0 \in U$, and let $\varphi(t), 0 \leq t<1$, be a continuous strictly positive function. Assume that

$$
f(0)=\frac{1}{c} \int_{U} f(\zeta) \varphi\left(|\zeta|^{2}\right) d m(\zeta)
$$

with $c=\int_{U} \varphi\left(|\zeta|^{2}\right) d m(\zeta)$ for all $\mathscr{M}$-harmonic functions in $\bar{U}$. Then $U$ is a ball centered at 0 . The only difference is that $\frac{1}{n} \log 1 /\left(1-|z|^{2}\right)$ must be replaced by $\Psi\left(|z|^{2}\right)$, the unique radial function vanishing at zero whose invariant Laplacian is $\varphi\left(|z|^{2}\right)\left(1-|z|^{2}\right)^{n+1}$. It is easy to check that

$$
\Psi^{\prime}(x)=h(x) g^{\prime}(x),
$$

where $h(x)=\int_{0}^{x} \varphi(t) t^{n-1} d t$. Then the equation $c g-\Psi=k$ has again at most two solutions, because $(c g-\Psi)^{\prime}=(c-h) g^{\prime}$ changes sign only once, $h$ being strictly increasing.

(c) In dimension $n=1$, the invariant Laplacian is $\left(1-|z|^{2}\right) D \bar{D}$; hence, the $\mathscr{M}$-harmonic functions are the usual harmonic functions in the plane. A Euclidean disk therefore has the mean-value property, with respect to the Lebesgue measure. In dimension $n>1$, by remark (b), the balls are the only domains having the property

$$
c f(0)=\int_{U} f(\zeta) d m(\zeta), \quad f \mathscr{M} \text {-harmonic in } \bar{U}, 0 \in U .
$$

The question arises whether this holds true for another point: what pairs $(a, U)$ $a \in U$ have the property

$$
c f(a)=\int_{U} f(\zeta) d m(\zeta), \quad c=m(U), f \mathscr{M} \text {-harmonic in } \bar{U} ?
$$

Does it follow that $a=0$ (and so $U$ is a ball)?

(d) The theorem in the case of harmonic function in $\mathbb{R}^{n}$ is still true if $U$ is of finite area but not necessarily bounded. In our case, is our theorem still true if $U$ is no more relatively compact in $B$ ?

\section{REFERENCES}

1. P. Ahern, M. Flores, and W. Rudin, An invariant volume-mean-value-property, J. Funct. Anal. 111 (1993), 380-397.

2. D. H. Armitage and M. Goldstein, The volume mean-value property of harmonic functions, Complex Variables 13 (1990), 185-193.

3. J. Bruna, A uniqueness theorem for invariantly harmonic functions in the unit ball of $\mathbb{C}^{n}$, Publ. Mat. 36 (1992), 421-426.

4. B. Epstein, On the mean value property of harmonic functions, Proc. Amer. Math. Soc. 13 (1962), 830.

5. Ũ. Kuran, On the mean-value property of harmonic functions, Bull. London Math. Soc. 4 (1972), 311-312.

6. B. Malgrange, Existence et approximation des solutions des equations aux derivees partielles et des equations de convolution, Ann. Inst. Fourier (Grenoble) 6 (1956), 271-355. 
7. W. Rudin, Function theory in the unit ball of $\mathbb{C}^{n}$, Grundlehren Math. Wiss., vol. 241, Springer-Verlag, New York, 1980.

8. H. Shapiro, private communciation, 1993.

Departament de Matemàtiques, Universitat Autònoma de Barcelona, 08193 BellaTERRA, BARCELONA, SPAIN

E-mail address: IMATD@CC.UAB.ES

U.E.R. de Mathematiques, Universite de Provence, 3, Place Victor Hugo, 13331, MarSEILle, France

E-mail address: DETRAZCCARLE2. UNIV-MRS.FR 